\title{
A Molecularly Imprinted Polymer-Based Technology for Rapid Testing of COVID-19
}

\author{
Trisita Nandy Chatterjee ${ }^{1}\left[\right.$ Rajib Bandyopadhyay $^{1}$
}

Received: 28 April 2020 / Revised: 3 June 2020 / Accepted: 9 June 2020 / Published online: 13 June 2020

C Indian National Academy of Engineering 2020

\begin{abstract}
The outbreak of COVID-19 has taken a large number of lives since 2019 and the death toll continues to increase all over the world. Recent data reports that about 27 lacs of people are infected with this virus till date and around 2 lacs are dead due to this pandemic. The situation in India is no way better. In India, almost all the states have become victim of this deadly pandemic. Considering the enormous population in India, citizens here are facing acute shortage of detection kits and many are dying even before the knowledge of their infection. The present treatise proposes a molecularly imprinted polymer (MIP) based technique for simple and rapid detection of COVID-19. The technique will be inexpensive, selective, reusable and easy to handle. It has been already implemented in our laboratory in order to detect the taste contributing agents found in tea. This article discusses the detailed methodology and the resultant analytical characteristic of the sensors developed so far and also outlines the suitability of the MIP technique towards fabrication of testing kits for rapid detection of COVID-19.
\end{abstract}

Keywords Coronavirus detection $\cdot$ Rapid testing kit $\cdot$ Molecularly imprinted polymer technique $\cdot$ Coronavirus specific aptamer

\section{Introduction}

The sudden outbreak of COVID-19 since 2019 have claimed a large number of lives and the count is increasing day by day. The infection emerged from Wuhan, China in the month of December, 2019 and has taken no time to drastically spread through several other countries across the globe namely, USA, Spain, Italy, France, UK, Germany, Turkey, Iran, Russia, Brazil, Belgium, Canada, Netherlands, Switzerland, India, Portugal, Peru, Ireland, Sweden, Saudi Arabia, Austria, Israel, Japan, etc. (https://edition.cnn.com/interactiv e/2020). Till date, above 27 lacs of citizens worldwide are affected by this pandemic. Though 7.5 lacs of them have recovered from this disease, above 1.9 lac persons have lost their lives already. The situation in India is also terrible. In this country above 23,000 citizens have been diagnosed with COVID-19 till date. Among them, above 4000 of the citizens

Trisita Nandy Chatterjee

trisita.chatterjee@gmail.com

1 Department of Instrumentation and Electronics Engineering, Jadavpur University, Salt Lake Campus, Block LB, Sector III, Plot 8, Salt Lake, Kolkata 700 098, India have recovered and 723 of them have lost their lives (https:// www.mygov.in/covid-19).

A number of reports have been published indicating the possible structure of corona virus and its linkages with previous pandemics in the last two decades like Severe acute respiratory syndrome corona virus (SARS-CoV) and Middle East respiratory syndrome coronavirus (MERS-CoV) (Zhou 2020; Zaki et al. 2012; Adhikari et al. 2020). Corona viruses (SARS-CoV-2) are enveloped single stranded RNA viruses that belongs to the genus of Betacoronavirus (Chan et al. 2020; $\mathrm{Lu}$ et al. 2020; Zhu et al. 2020). From the phylogenetic analysis, researchers have revealed that SARS-CoV-2 is closely related to two bat-derived SARS-like coronaviruses, namely bat-SL-CoVZC45 (GenBank accession no. MG772933.1) and bat-SL-CoVZXC21 (GenBank accession no. MG772934.1), but its resemblance is less likely similar to that of SARS-CoV and MERS-CoV. SARS-CoV-2 can be transmitted from one human being to another and the person having the carrier can transmit up to three other people. The symptoms associated with this disease usually takes $2-5$ days to become visible. The initial symptoms of COVID-19 resemble mostly those of common cold and flu. These are mainly, fever, tiredness, cough and aches. In due course of time, the severity of COVID-19 may cause pneumonia, breathing difficulty, infection in the lower 
respiratory tract, diarrhoea, etc. (Zhou et al. 2020; Li et al. 2020; Udugama et al. 2020). Moreover, many infected patients have been reported to be asymptomatic as well.

Consequent upon the spread of the disease as an epidemic, firstly, it is very important to rapidly identify the person bearing the same so that treatment can be started instantly in case he is positively suspected. Recent guidelines as published by the Chinese government suggests that the diagnosis of COVID-19 can be ascertained by gene sequencing for respiratory or blood samples which serves as a key indicator for reverse transcription polymerase chain reaction (RT-PCR) (Lai et al. 2020; Chen et al. 2020). A large number of RT-PCR kits have been designed by different countries in order to detect SARS-CoV-2. According to published reports, RT-PCR can be performed by means of either a one-step or a two-step assay. But these assay techniques are either difficult to optimize or time consuming (Chang et al. 2020). Moreover, there are not adequate number of PCR reagent kits available. COVID-19 can also be screened by means of Computed Tomography (CT) scans. Automated CT image based on Artificial Intelligence (AI) have been employed for the detection, quantification and monitoring of COVID-19 (Gozes et al. 2020). In a study by Fei et al., a deep learning-based system has been developed for automatic segmentation of all lung and infection sites using chest CT (Shan et al. 2020). Pulmonary CT images accompanied with deep learning techniques have been also used by Xiaowei et al. inorder to establish an early screening model for isolating COVID-19 pneumonia and InfluenzaA viral pneumonia from healthy cases (Xu et al. 2020). In a study by Shuai et al., a deep learning method has been employed based on the COVID-19 radiographic changes from CT images. It can extract the graphical features of COVID-19 to provide clinical diagnosis before pathogenic testing and thus save critical time for the disease diagnosis (Wang et al. 2020). But these techniques suffer from certain disadvantages, as CT scan based imaging are expensive, require skilled experts and also cannot selectively diagnose COVID-19 in a single shot (Narin et al. 2020).

The present treatise proposes a molecularly imprinted polymer (MIP) based technique for simple and rapid detection of COVID-19. The proposed technique will be inexpensive, selective, reusable and easy to handle. Here, we are proposing the application of a corona virus specific aptamer as the recognition element for binding with the polymer matrix selected and optimized from a library by repeated experimental trials.

\section{Molecularly Imprinted Polymer Technique: Principles and Applications}

Molecularly imprinted polymer (MIP) based techniques are highly advantageous in the field of sensing due to its excellent ruggedness, thermal, chemical and mechanical stabilities. MIPs are synthetic receptors which have recognition sites; being complementary to the shape and orientation of the target molecule. These are very useful because of their unique pre-determinative characteristics, specific and practical abilities for template molecule recognition (Yang et al. 2011). The principle involves the synthesis of MIPs with molecular recognition cavities that have specific selectivity for the template molecules (Yuan et al. 2016; Motaharian et al. 2016; Cheng et al. 2016).

In our laboratory, we have used this method in order to develop sensors that can specifically detect theaflavin (TF), catechin (CAT), epigallocatechin-3-gallate (EGCG) and caffeine (CAF), respectively (Chatterjee et al. 2017, 2018, 2019; Das et al. 2020). A schematic of the MIP based technique for the detection of TF is depicted in Fig. 1.

Theaflavin holds a special significance as it is highly responsible for its astringency and also contribute to the bright orange-red colour of black tea. Catechin and epigallocatechin-3-gallate, on the other hand, are the equally important polyphenols present in green tea. These are also responsible for the astringency and antioxidant nature of green tea. For the detection of TF, an extremely simple and cost effective synthesis route has been employed to develop an electrode from a composite of graphite and acrylamide-divinylbenzene (AM-DVB) copolymer. The sensor demonstrated an excellent sensitivity and selectivity to TF. Moreover, the electrodes are also repeatable and reproducible in nature. In regard to the detection of CAT, acrylonitrile has been optimized as the monomer due to the presence of highly polar nitrile group that are capable of making hydrogen bonds with the template molecule. Moreover, acrylonitrile also increases the mechanical strength and ruggedness of the graphite based composite. For the detection of EGCG, an MIP electrode has been synthesized and further modified with nanostructured nickel hydroxide nanopetals using a simple solution route, thereby resulting

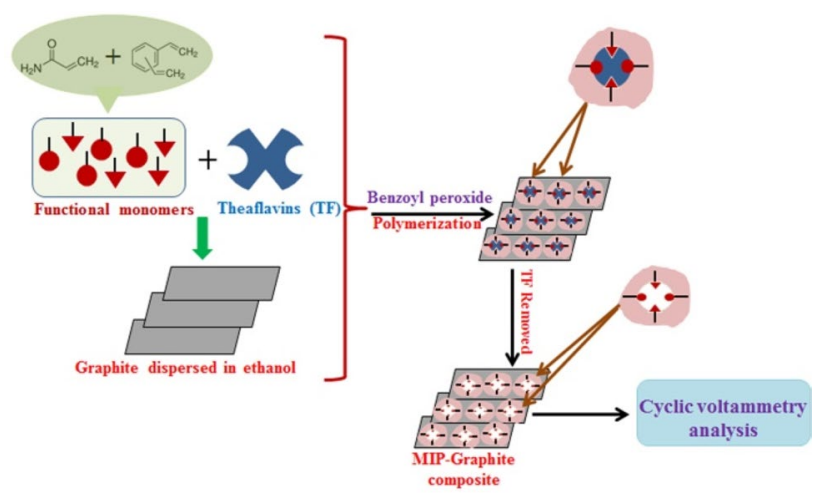

Fig. 1 Detection principle of TF using MIP technique (Chatterjee et al. 2017) 
into MIP-Ni $(\mathrm{OH})_{2}$ electrode. The analytical characteristic of the electrode has been studied in detail and it delivered a considerable wide linear range along with good selectivity, repeatability, reproducibility and stability characteristics. Further, CAF molecule has been also detected successfully by using the principle of MIP. Herein, the sensing material has been developed using the co-polymer of acrylonitrile (AN) and ethylene glycol dimethacrylate (EGDMA) followed by impregnation of $\mathrm{TiO}_{2}$ nanocubes into it. The analytical characteristics revealed acceptable selectivity, repeatability, stability and reproducibility of the electrode.

The real time performance of all the sensors in presence of black tea/green tea samples have been validated with that of standard high performance liquid chromatography (HPLC) technique. Both the results were correlated using partial least square regression (PLSR)/principal component regression (PCR) algorithms. The analytical characteristics and the prediction accuracies of all the developed sensors have been listed in Table 1.

The sensors discussed previously, were synthesized using the solution route and electrodes were prepared by pressing the powder sample into cylindrical glass tubes of inner diameter $2.5 \mathrm{~mm}$. One end of the tube was connected with Pt wire in order to establish electrical contacts. In another work, we have applied the principle of MIP on screen printed carbon electrodes (SPCEs) in order to detect chlorpyrifos (CP) in lower concentration levels (Chatterjee et al. 2019). The SPCEs were coated with cobalt oxide $\left(\mathrm{Co}_{3} \mathrm{O}_{4}\right)$ nanoparticles and polymerized using pyrrole for the fabrication of the MIP based sensing material. In its first phase of development, the sensor exhibited a linear range from 0.1 to $100 \mathrm{ppb}$ of $\mathrm{CP}$ and was also found to be selective in nature.

\section{Rapid Detection of COVID-19 Using the Principle of MIP}

In view of the prevailing circumstances worldwide, it is well evident that a relatively inexpensive, easy to use and point of care testing kit is in high demand for the detection of COVID-19. This is because, rapid detection of the pandemic can only result into early treatments of the infected patients. Considering the selectivity and the sensitivity of the MIP based sensors, these types of material can also be used to detect SARS-CoV-2. Our limited literature survey indicates that previously recognition of Zika virus, Ebola virus or Norovirus were made possible by selecting their DNA or virus specific aptamers as recognition element, respectively (Afsahi et al. 2018; Kaushik et al. 2018; Song et al. 2016; Lee et al. 2018; Weerathunge et al. 2019; Saylan et al. 2019). Towards this direction, detection of SARS-CoV-2 (corona virus) can also be performed by selecting appropriate polymer from a library and using corona virus specific aptamer as the recognition element. For the development of sensing materials, polymers belonging to the acrylic group like acrylamide, acrylic acid, methyl acrylate, methyl methacrylate, ethyl acrylate and their mixtures can be selected. They will be further reinforced on graphene or conducting metal oxides for providing enhanced conductivity. The best performing polymer-conducting material nanocomposite will be selected based on repeated experimentations. The process of imprinting and subsequent removal of the template can be performed following the typical schematic as shown in Fig. 1. The resultant sensor will be embedded with an electronic circuitry fitted with an alarm such that the user can be immediately informed of his health status.

\section{Conclusions}

It is well evident that the present situation demands the development of rapid testing kits for the detection of COVID-19 as early as possible. The kit should also comply well with the results of the clinical trials before being procured into the market. Considering the need of the hour, in this study we have proposed a molecularly imprinted polymer based method in order to detect COVID 19 in a simple

Table 1 Typical performance matrix of the developed sensing materials

\begin{tabular}{|c|c|c|c|c|c|c|c|}
\hline Sl. no. & Electrode & Precursors & Linear range $(\mu \mathrm{M})$ & LOD & $\begin{array}{l}\text { Repeatabil- } \\
\text { ity (\% RSD) }\end{array}$ & $\begin{array}{l}\text { Reproduc- } \\
\text { ibility (\% } \\
\text { RSD) }\end{array}$ & $\begin{array}{l}\text { Average prediction } \\
\text { accuracy in tea samples } \\
(\%)\end{array}$ \\
\hline 1 & MIP-TF & $\begin{array}{l}\text { Monomer: Acrylamide } \\
\text { Crosslinker: DVB }\end{array}$ & $20-100$ & $14 \mu \mathrm{M}$ & 5.30 & 6.76 & PLSR: 94 \\
\hline 2 & MIP-CAT & $\begin{array}{l}\text { Monomer: Acrylonitrile } \\
\text { Crosslinker: EGDMA }\end{array}$ & $5-100$ & $37 \mathrm{nM}$ & 4.14 & 5.95 & PLSR: 92 \\
\hline 3 & MIP-EGCG & $\begin{array}{l}\text { Monomer: Acrylonitrile } \\
\text { Crosslinker: EGDMA } \\
\text { Modifier: } \mathrm{Ni}(\mathrm{OH})_{2} \text { nanoparticles }\end{array}$ & $10-100$ & $7 \mathrm{nM}$ & 2.17 & 6.45 & PLSR: 93.85 \\
\hline 4 & MIP-CAF & $\begin{array}{l}\text { Monomer: Acrylonitrile } \\
\text { Crosslinker: EGDMA } \\
\text { Modifier: } \mathrm{TiO}_{2} \text { nanoparticles }\end{array}$ & $5-120$ & $0.6 \mu \mathrm{M}$ & 2.08 & 5.49 & $\begin{array}{l}\text { PLSR: } 92.94 \\
\text { PCR: } 93.75\end{array}$ \\
\hline
\end{tabular}


and effective manner. Towards this direction, some of our previous works using this principle have been elucidated and the consequent performances of the sensing materials have also been highlighted. It is interesting to note that if the preparation of sensing materials can be made based on thin film techniques, the corresponding detection limit can be lowered even to $\mathrm{ppm} / \mathrm{ppb}$ levels. The prototype after complete development can be subjected to extensive clinical trials in collaboration with reputed medical institutes followed by commercialization.

Funding Not applicable.

Availability of data and material Literature survey and previous publications of self.

\section{Compliance with ethical standards}

Conflict of interest The authors declare no conflicts of interests for this work.

Code availability Not applicable.

\section{References}

Adhikari SP et al (2020) Epidemiology, causes, clinical manifestation and diagnosis, prevention and control of corona virus disease (COVID-19) during the early outbreak period: a scoping review. Infect Dis Pov 9:1-12

Afsahi S et al (2018) Novel graphene-based biosensor for early detection of Zika virus infection. Biosens Bioelectron 100:85-88

Chan JF et al (2020) Genomic characterization of the 2019 novel human-pathogenic coronavirus isolated from a patient with a typical pneumonia after visiting Wuhan. Emerg Microbes Infect 9:221-236

Chang D et al (2020) Epidemiologic and clinical characteristics of novel coronavirus infections involving 13 patients outside Wuhan, China. JAMA 323:1092-1093

Chatterjee TN et al (2019) A molecularly imprinted polymer conjugated cobalt oxide nanoparticle based screen printed sensor for enhanced sensing of chlorpyrifos. In: 2019 IEEE international symposium on olfaction and electronic nose (ISOEN). https://doi. org/10.1109/isoen45098.2019

Chatterjee TN et al (2017) Detection of theaflavins in black tea using a molecular imprinted polyacrylamide-graphite nanocomposite electrode. Sens Act B 246:840-847

Chatterjee TN et al (2018) Molecular imprinted polymer based electrode for sensing catechin $(+C)$ in green tea. IEEE Sens $J$ $18: 2236-2244$

Chatterjee TN et al (2019) Development of a nickel hydroxide nanopetal decorated molecularly imprinted polymer based electrode for sensitive detection of epigallocatechin-3-gallate in green tea. Sens Act B 283:69-78

Chen N et al (2020) Epidemiological and clinical characteristics of 99 cases of 2019 novel coronavirus pneumonia in Wuhan, China: a descriptive study. Lancet 395:507-513

Cheng Y et al (2016) Preparation of a molecularly imprinted fluorescent chemosensor using quinoline modified vinyl- $\beta$-cyclodextrin and acrylamide as monomers for the selective recognition of spermidine. Anal Methods 8:5838-5842
Das D et al (2020) Titanium oxide nanocubes embedded molecularly imprinted polymer-based electrode for selective detection of caffeine in green tea. IEEE Sens J 20:6240-6247

Gozes O et al (2020) Rapid AI development cycle for the coronavirus (COVID-19) pandemic: initial results for automated detection \& patient monitoring using deep learning CT image analysis, pp 1-19. arXiv preprint arXiv:2003.05037

Kaushik A et al (2018) A sensitive electrochemical immunosensor for label-free detection of Zika-virus protein. Sci Rep 8:9700-9705

Lai $C$ et al (2020) Severe acute respiratory syndrome coronavirus 2 (SARS-CoV-2) and coronavirus disease-2019 (COVID-19): the epidemic and the challenges. Int J Antimicrob Agents 55:1-9

Lee J et al (2018) A multi-functional gold/iron-oxide nanoparticle-CNT hybrid nanomaterial as virus DNA sensing platform. Biosens Bioelectron 102:425-431

Li X et al (2020) Potential of large "first generation" Human-to-human transmission of 2019-nCoV. J Med Virol 92:448-454

Lu R et al (2020) Genomic characterization and epidemiology of 2019 novel coronavirus: implications of the virus origins and receptor binding. Lancet. https://doi.org/10.1016/S0140-6736(20)30251-8

Motaharian A et al (2016) Molecularly imprinted polymer nanoparticles-based electrochemical sensor for determination of diazinon pesticide in well water and apple fruit samples. Anal Bioanal Chem 408:6769-6779

Narin A et al (2020) Automatic detection of coronavirus disease (COVID-19) using X-ray images and deep convolutional neural networks. arXiv:2003.10849

Saylan Y et al (2019) An alternative medical diagnosis method: biosensors for virus detection. Biosensors 9:1-22

Shan F et al (2020) Lung infection quantification of COVID-19 in CT images with deep learning, pp 1-19. arXiv preprint arXiv :2003.04655

Song J et al (2016) Instrument-free point-of-care molecular detection of Zika virus. Anal Chem 88:7289-7294

Udugama B et al (2020) Diagnosing COVID-19: the disease and tools for detection. ACS Nano. https://doi.org/10.1021/acsnano.0c026 24

Wang S et al (2020) A deep learning algorithm using CT images to screen for Corona Virus Disease (COVID-19). medRxiv preprint, pp 1-26. https://doi.org/10.1101/2020.02.14.20023028

Weerathunge $P$ et al (2019) Ultrasensitive colorimetric detection of murine norovirus using NanoZyme aptasensor. Anal Chem 91:3270-3276

$\mathrm{Xu}$ X et al (2020) Deep learning system to screen coronavirus disease 2019 pneumonia. arXiv preprint arXiv:2002.09334, pp 1-29

Yang Y et al (2011) Molecularly imprinted polymer on carbon microsphere surfaces for adsorbing dibenzothiophene. Colloids Surf A Physicochem Eng Asp 377:379-385

Yuan Y et al (2016) Preparation of core-shell magnetic molecular imprinted polymer with binary monomer for the fast and selective extraction of bisphenol A from milk. J Chromatogr A 1462:2-7

Zaki AM et al (2012) Isolation of a novel coronavirus from a man with pneumonia in Saudi Arabia. N Engl J Med 367:1814-1820

Zhou P et al (2020) Discovery of a novel coronavirus associated with the recent pneumonia outbreak in humans and its potential bat origin. bioRxiv. https://doi.org/10.1101/2020.01.22.914952

Zhou P et al (2020) A pneumonia outbreak associated with a new coronavirus of probable bat origin. Nature 579:270-273

Zhu N et al (2020) A novel coronavirus from patients with pneumonia in China. N Engl J Med. https://doi.org/10.1056/NEJMoa2001017

Publisher's Note Springer Nature remains neutral with regard to jurisdictional claims in published maps and institutional affiliations. 